\title{
Murine nasal septa for respiratory epithelial air-liquid interface cultures
}

\author{
Marcelo B. Antunes1, Bradford A. Woodworth1, Geeta Bhargave1, Guoxiang \\ Xiong1, Jorge L. Aguilar², Adam J. Ratner², James L. Kreindler³, Ronald C. \\ Rubenstein ${ }^{1}$, and Noam A. Cohen ${ }^{1}$ \\ ${ }^{1}$ University of Pennsylvania, Philadelphia, PA, ${ }^{2}$ Columbia University, New York, NY, \\ and ${ }^{3}$ University of Pittsburgh, Pittsburgh, PA, USA
}

BioTechniques 43:195-204 (August 2007)

doi 10.2144/000112493

\begin{abstract}
Air-liquid interface models using murine tracheal respiratory epithelium have revolutionized the in vitro study of pulmonary diseases. This model is often impractical because of the small number of respiratory epithelial cells that can be isolated from the mouse trachea. We describe a simple technique to harvest the murine nasal septum and grow the epithelial cells in an air-liquid interface. The degree of ciliation of mouse trachea, nasal septum, and their respective cultured epithelium at an air-liquid interface were compared by scanning electron microscopy (SEM). Immunocytochemistry for type IV $\beta$-tubulin and zona occludens-1 (Zo-1) are performed to determine differentiation and confluence, respectively. To rule out contamination with olfactory epithelium $(O E)$, immunocytochemistry for olfactory marker protein $(O M P)$ was performed. Transepithelial resistance and potential measurements were determined using a modified vertical Ussing chamber. SEM reveals approximately $90 \%$ ciliated respiratory epithelium in the nasal septum as compared with $35 \%$ in the mouse trachea. The septal air-liquid interface culture demonstrates comparable ciliated respiratory epithelium to the nasal septum. Immunocytochemistry demonstrates an intact monolayer and diffuse differentiated ciliated epithelium. These cultures exhibit a transepithelial resistance and potential confirming a confluent monolayer with electrically active airway epithelium containing both a sodium-absorptive pathway and a chloride-secretory pathway. To increase the yield of respiratory epithelial cells harvested from mice, we have found the nasal septum is a superior source when compared with the trachea. The nasal septum increases the yield of respiratory epithelial cells up to 8 -fold.
\end{abstract}

\section{INTRODUCTION}

The development of primary culture models of transgenic mouse tracheal epithelial cells has greatly facilitated the study of human respiratory diseases. These primary culture models are confluent, fully differentiated, ciliated respiratory epithelium at an air-liquid interface on a semipermeable membrane that mimic many characteristics of murine tracheal epithelial cells in vivo. Prior to the development of murine models, suboptimal methods involving non-polarized and sometimes poorly differentiated primary cultures and immortalized cell lines were used for respiratory epithelial cell research. These models made logistical sense because they often supplied copious numbers of cells through expansion and passage of the cells. While the murine air-liquid interface models are ideal for the development of differentiated respiratory epithelial cells, expansion and passage of these cells will ultimately decrease the ability for these cells to differentiate (1). Therefore, one of the primary limitations of in vitro murine models includes the large number of mice required to obtain a significant number of tracheal epithelial cells. Typically, dissociation of respiratory epithelium from two mouse tracheas is required for the development of an air-liquid interface on one transwell membrane (6.5-mm diameter). This can be cost-prohibitive when attempting to develop in vitro airway models of transgenic mice. An additional source of respiratory epithelium will increase the utility of these mice and, at the same time, decrease expenses.

Our recent investigations have found that the nasal septum is a superb source of murine respiratory epithelium. Most research involving murine nasal septa has primarily focused on olfaction, so the potential for respiratory cell culture has largely been ignored. The two sides of the murine nasal septum are covered with mucosa, and the overall surface area is much larger in comparison to the mouse trachea. The olfactory system of the mouse has four anatomically distinct chemosensory areas on the nasal septum. (Figure 1) The main olfactory epithelium (OE), the septal organ of Masera (SO), and the vomeronasal organ (VNO) have bipolar sensory neurons that reside in a pseudostratified neuroepithelium. The MOE and SO are part of the main olfactory system, which primarily detects odorant molecules, while the VNO detects pheromones and is the major component of the accessory olfactory system $(2,3)$. The fourth anatomically distinct chemosensory area of the nasal septum is the septal organ of Grüneberg (SOG) (4). This chemosensory area is submucosal in location and covered with respiratory epithelium. Approximately $50 \%$ of the total surface area of the septum is respiratory epithelium. It has been presumed that nasal respiratory epithelium is very similar to tracheal respiratory epithelium in structure and function. Furthermore, sinonasal disorders mimic or coexist with many respiratory diseases, such as cystic fibrosis, aspirin-sensitive asthma with polyps, and allergic fungal sinusitis (the upper airway correlates to allergic bronchopulmonary aspergillosis) (5). Thus we have focused our efforts on establishing airliquid interface cultures from the mouse nasal septum and demonstrate that our technique increases the yield of respiratory epithelium 8-fold. Additionally, our technique for removing the nasal septum is simple and straightforward.

\section{MATERIALS AND METHODS}

\section{Tissue Culture Technique}

Harvest of mouse nasal septum. Following euthanasia with a $\mathrm{CO}_{2}$ gas chamber and cervical dislocation, the mouse was placed on a Styrofoam ${ }^{\circledR}$ dissection table in the prone position and secured with several 18-gauge needles. The skin at the nape of the 


\section{Short Technical Reports}

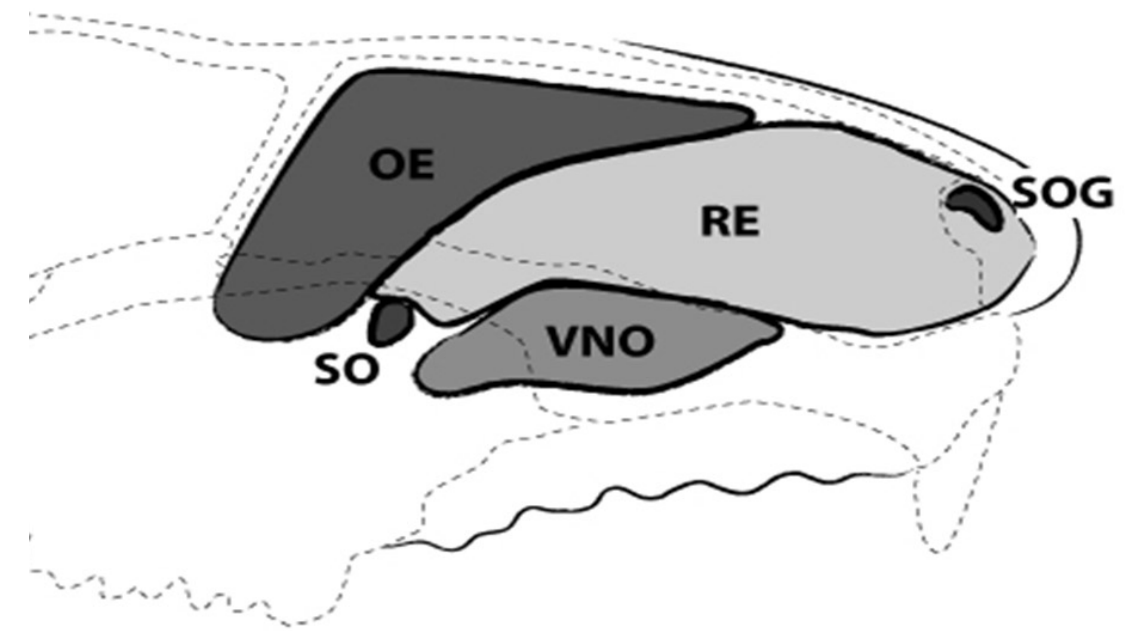

Figure 1. The distribution of respiratory and olfactory epithelium (OE) on the murine nasal septum. The septum contains the main OE, the septal organ of Masera (SO), the vomeronasal organ (VNO), and the septal organ of Grüneberg (SOG), which is submucosal and covered with respiratory epithelium. Note that approximately $50 \%$ of the surface area is respiratory epithelium on both sides of the septum.

neck was incised with fine dissecting scissors, and the incision rotated around the entire neck. This skin was then dissected anteriorly and completely removed, exposing the bone over the entire skull and nose. The skull was then sectioned in the coronal plane posterior to the eyes (Figure 2A, cut no. 1). The remnant of the brain was completely removed, leaving the anterior aspect of the skull base exposed. The most anterior point of the skull base on either side is directly posterior to the mouse nasal cavities. The remaining portion of the skull was removed to the posterior aspect of the nasal cavity (Figure 2A, cut no. 2).

The dorsum of the mouse nose has two lines representing embryonic fusion planes formed between the two maxillas (laterally) and the ethmoid bone (medially). Using this as a guide, a scissor was inserted into the posterior aspect of the nasal cavity, and the suture line was incised separating one side of the septum (medially) from the maxilla (laterally) (Figure 2, A and B, cut no. 3). The scissor was then turned inferiorly to section the palate, and thus completely separated the septum from the lateral nasal wall. The procedure was then repeated on the other side (Figure 2, $\mathrm{A}$ and $\mathrm{C}$, cut no. 4). The remaining attachment at the nasal tip was severed and the septum completely removed.
Coating semipermeable support membranes. The tissue culture insert semipermeable support membranes $\left(\right.$ Costar $^{\circledR}$ Transwell ${ }^{\circledR}$ clear 24-well plate inserts, $0.4-\mu \mathrm{m}$ pore; Corning Life Sciences, Lowell, MA, USA) were coated with $100 \mu \mathrm{L} 50 \mu \mathrm{g} / \mathrm{mL}$ human placental collagen (type VI; Rockland Immunochemicals, Gilbertsville, PA, USA) using a sterile technique $24-48 \mathrm{~h}$ prior to tissue harvest. The inserts were incubated in a $37^{\circ} \mathrm{C}$ biosafety incubator over $24 \mathrm{~h}$; the collagen solution was removed, and the inserts were washed twice with phosphate-buffered saline (PBS) before use.

Culture of septal respiratory epithelial cells. Mouse air-liquid interface (ALI) cultures were adapted from previously published methods (6). After isolation of the mouse septum, it was placed temporarily in a 50-mL conical tube containing either PBS or a 1:1 mixture of Dulbecco's modified Eagle's medium (DMEM), Nutrient Mixture Ham's F-12 medium (Invitrogen, Carlsbad, CA, USA), 100 $\mathrm{IU} / \mathrm{mL}$ penicillin, and $100 \mu \mathrm{g} / \mathrm{mL}$ streptomycin if a long dissection is anticipated. Upon finishing the dissection of all harvested mouse septa, they were transferred into $20-\mathrm{mL}$ volumes of dissociation media containing minimal essential medium (MEM; Invitrogen), penicillin $(60 \mathrm{IU} / \mathrm{mL})$-streptomycin $(60$ $\mu \mathrm{g} / \mathrm{mL}$ ), $1.4 \mathrm{mg} / \mathrm{mL}$ Pronase (Sigma-
Aldrich, St. Louis, MO, USA), and 0.1 $\mu \mathrm{g} / \mathrm{mL}$ DNase (Roche Applied Science, Indianapolis, IN, USA). We generally place up to eight mouse septa per 20 $\mathrm{mL}$ dissociation media. This media was prewarmed in a $5 \% \mathrm{CO}_{2}$ chamber at $37^{\circ} \mathrm{C}$ for $1 \mathrm{~h}$ prior to use with the cap loosely fitted to allow for diffusion of $\mathrm{CO}_{2}$ into the media. The septa were incubated in the dissociation media in the $5 \% \mathrm{CO}_{2}$ chamber at $37^{\circ} \mathrm{C}$ for $1 \mathrm{~h}$. To stop the enzymatic dissociation, 5 $\mathrm{mL}$ sterile $5 \%$ fetal bovine serum (FBS; HyClone, Logan, UT, USA) were added, followed by a further 2-min incubation. The epithelial cells were dissociated by gentle agitation of the sinonasal tissue, achieved by 12 inversions of the tube.

The tissue was removed from the suspension and transferred to $10 \mathrm{~mL}$ culture media consisting of a 1:1 mixture

A
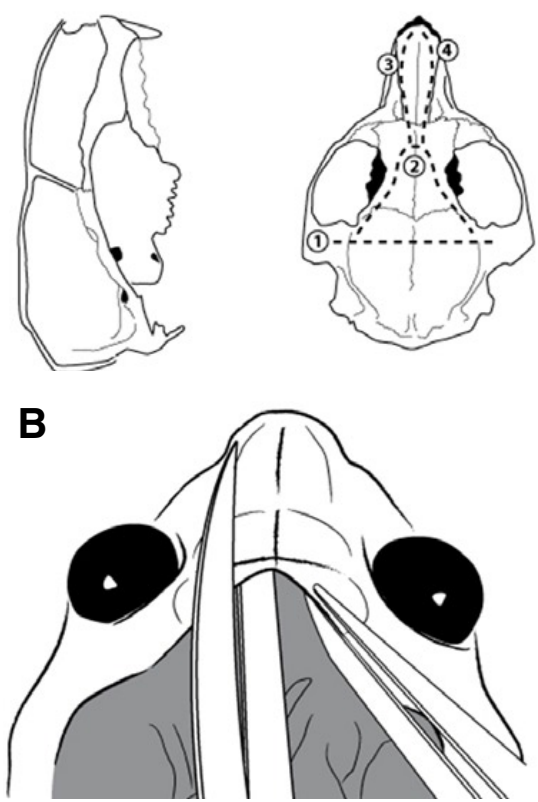

Figure 2. Harvesting the mouse septum. (A) Cut no. 1: the skin is dissected and completely removed from the skull and nose, and the skull sectioned in the coronal plane. Cut no 2: next, the remainder of the anterior skull is removed. The posterior aspect of the skull base has been removed and the scissor inserted into the posterior nasal cavity. Cut no. 3: the suture line is then incised bilaterally revealing the nasal septum and the oral tongue below (B) The scissor will separate the two maxillas (laterally) and the ethmoid bone (medially) along the distinct suture line. (C) Cut no. 4: the upper palate and anterior nasal tip are removed next to complete the dissection. The structure of the bony septum is shown in panel A (left). 
of DMEM and Nutrient Mixture Ham's F-12 medium containing penicillin (100 $\mathrm{IU} / \mathrm{mL})$-streptomycin $(100 \mu \mathrm{g} / \mathrm{mL}), 5 \%$ heat-inactivated FBS, and $120 \mathrm{IU} / \mathrm{mL}$ ITS $^{\text {TM }}$ universal culture supplement (BD Biosciences, Bedford, MA, USA). This tube was gently inverted 12 times, as before, to release further epithelial cells. The tissue was removed from this suspension, and the two resultant cell suspensions were pooled and centrifuged at $120 \times g$ for $5 \mathrm{~min}$ at room temperature $\left(21^{\circ}-23^{\circ} \mathrm{C}\right)$. The supernatant was removed, and the cell pellet was gently resuspended and washed in $10 \mathrm{~mL}$ culture media, centrifuged at $120 \times g$ for $5 \mathrm{~min}$ at room temperature and then resuspended in $5 \mathrm{~mL}$ culture media. This suspension was incubated at $37^{\circ} \mathrm{C}$ for $2 \mathrm{~h}$ in $100 \mathrm{~mm}$ Primaria $^{\mathrm{TM}}$ culture dishes (BD Biosciences) to remove the nonepithelial cells. Approximately one septum per Primaria dish is needed to efficiently reduce fibroblast contamination. Any visible undigested tissue fragments were removed, after which the cell suspension containing the nonadherent cells was collected with a fine-tip pipet. Next, the suspension was centrifuged at $120 \times g$ for $5 \mathrm{~min}$ at room temperature. The cell pellet was gently resuspended in culture media and mixed with a fine-tip pipet. The cell yield was then counted using a hemocytometer. The cell yield for one trachea using this technique is approximately $2 \times 10^{5}$ cells. The cell yield for one nasal septum is approximately 1.5 $\times 10^{6}$ for one septum.

The dissociated cells were seeded at a density of $4 \times 10^{5}$ cells per semipermeable support membrane in $200 \mu \mathrm{L}$ culture media, with $600 \mu \mathrm{L}$ culture media outside the insert in the basal compartment. The cells were incubated at $37^{\circ} \mathrm{C}$ in $5 \% \mathrm{CO}_{2}$ in a humidified incubator for 3 days. On day four, the medium on the apical surface was removed along with any nonadherent cells and debris. The basal medium was replaced with $600 \mu \mathrm{L}$ differentiation media consisting of a 1:1 mixture of DMEM and Nutrient Mixture Ham's F-12 medium containing $100 \mathrm{IU} / \mathrm{mL}$ penicillin, $100 \mu \mathrm{g} / \mathrm{mL}$ streptomycin, and $2 \%$ NuSerum $^{\mathrm{TM}}$ (BD Biosciences). The basal medium was replaced twice weekly. Primary cultures grown at an air-liquid interface in this manner reach maximal confluency at 1 week on approximately $80 \%-90 \%$ of the permeable support membrane plated at the above concentration. Maximal differentiation (generation of cilia), however, is achieved at 3 weeks. Cilia begin to appear between 1 and 2 weeks. Depending on the contamination of nonepithelial cells, generally $80 \%$ of the surface will have differentiated cilia by 2 weeks. One nasal septum yields approximately four air-liquid interface cultures. In general, we use 6- to 10week-old female Balb/c or C57 mice. We have not found a difference in yield or characteristics of the cultures between these two strains of mice, although we did not investigate the ideal age for optimizing yield; waiting until the mice have grown to adult size will likely increase the number of epithelial cells due to a larger septal area.

\section{Scanning Electron Microscopy}

SEM was performed on a mouse nasal septum, trachea, and air-liquid interface cultures at 17 days. The formalin-fixed mouse trachea, septal mucosa, and air-liquid interface membranes were dehydrated in a progression of increasing ethanol concentrations, up to $100 \%$ ethanol. The specimens were then critical-point dried in $\mathrm{CO}_{2}$, mounted on scanning electron microscope stubs, and sputtercoated with gold palladium to a depth of $12 \mathrm{~nm}$. The surface of the trachea and septum were then examined with an AMR-1400 scanning electron microscope at an accelerating voltage of 20 $\mathrm{kV}$. Representative photomicrographs were taken at various angles to effectively display the specimen so that any error in assessment is minimized due to the tilt of the specimen or other artifact. Photomicrographs were evaluated for the percentage of ciliated epithelium in the mouse trachea, nasal septum, and air-liquid interface monolayer.

\section{Immunocytochemistry}

Localization by immunocytochemistry of cilia (type IV $\beta$-tubulin) and tight junctions (Zo-1) was performed on cultured monolayers in transwell inserts to confirm differentiation and confluence, respectively. Mouse anti- human type IV $\beta$-tubulin monoclonal antibodies and rabbit anti-human Zo-1 polyclonal antibodies were obtained from Invitrogen. Negative controls were performed in parallel without a primary antibody incubation step. Nonspecific staining was blocked with $5 \%$ goat serum and $1 \%$ bovine serum albumin (BSA). The cells were permeabilized with $0.3 \%$ Triton $^{\circledR} \mathrm{X}$ 100 and incubated in primary antibody (type IV $\beta$-tubulin, 1:500; Zo-1, 1:100) overnight at $4^{\circ} \mathrm{C}$. After three washes with PBS, the transwell insert was incubated in fluorescein isothiocyanate (FITC)-coupled goat anti-mouse immunoglobulin $\mathrm{G}(\mathrm{IgG} ; 1: 500)$ and rhodamine-coupled goat anti-rabbit IgG (1:500) at room temperature for $90 \mathrm{~min}$. The membranes were washed three times in $1 \times$ PBS and then cut from the plastic support mold. They were mounted with Gel Mount ${ }^{\mathrm{TM}}$ aqueous mounting medium (Sigma-Aldrich) on a glass slide. The slides were then imaged on a Zeiss LSM510META confocal microscope.

Because OE comprises approximately half of the nasal septum, cultured epithelial monolayers were incubated with OMP antibodies (specific to OE) and type IV $\beta$-tubulin (specific to respiratory cilia). Septal olfactory mucosa was harvested and fixed for positive control. Mouse septal air-liquid interface cultures were incubated in primary antibodies (type IV $\beta$-tubulin, 1:500; OMP, 1:1000) overnight at $4{ }^{\circ} \mathrm{C}$. After three washes with PBS, the transwell insert was incubated in FITC-coupled goat antirabbit $\operatorname{IgG}(1: 500)$ and rhodaminecoupled goat anti-mouse IgG (1:500) at room temperature for $90 \mathrm{~min}$. The remainder of the protocol was performed in an identical fashion to our previous methods, with the exception that the slides were counterstained with 4',6-diamidino-2-phenylindole (DAPI) nuclear stain.

\section{Electrophysiology}

Solutions and chemicals. The bath solution contained $120 \mathrm{mM} \mathrm{NaCl}, 25$ $\mathrm{mM} \mathrm{NaHCO} 3,3.3 \mathrm{mM} \mathrm{KH}_{2} \mathrm{PO}_{4}, 0.8$ $\mathrm{mM} \mathrm{K}_{2} \mathrm{HPO}_{4}, 1.2 \mathrm{mM} \mathrm{MgCl} 2,1.2 \mathrm{mM}$ $\mathrm{CaCl}_{2}$, and $10 \mathrm{mM}$ glucose. The $\mathrm{pH}$ of this solution is 7.3-7.4 when gassed 
with a mixture of $95 \% \mathrm{O}_{2}-5 \% \mathrm{CO}_{2}$ at $37^{\circ} \mathrm{C}$. Chemicals were obtained from Sigma-Aldrich. Each chemical was made as a $1000 \times$ stock and used at $1 \times$ in the Ussing chamber. Amiloride was made as an aqueous solution. Forskolin and glybenclamide were dissolved in dimethyl sulfoxide (DMSO).

Short circuit measurements. Transwell inserts (Corning Life Sciences) were mounted in a modified, vertical Ussing chamber, and the monolayers were continuously shortcircuited after fluid resistance compensation using automatic voltage clamps (VCC 600; Physiologic Instruments, San Diego, CA, USA). Transwell filters were mounted in bath solution warmed to $37^{\circ} \mathrm{C}$, and the solution was continuously gas-lifted with a $95 \% \mathrm{O}_{2}$ $5 \% \mathrm{CO}_{2}$ mixture. The Ussing chambers were placed on heated stage, but were not mounted in a jacketed holder. The short circuit current $\left(\mathrm{I}_{\mathrm{SC}}\right)$ was digitized at one sample per second, and data were stored on a computer hard drive using Acquire \& Analyze software build 2.2 (Physiologic Instruments). Transepithelial resistance $\left(R_{T}\right)$ was measured every $60 \mathrm{~s}$ by passing a 2-mV, 2-s, bipolar pulse across the monolayer and calculating $R_{T}$ by Ohm's law $(V=I R)$. By convention, a positive deflection in $\mathrm{I}_{\mathrm{SC}}$ is defined as the net movement of a cation in the serosal to mucosal direction.

\section{RESULTS AND DISCUSSION}

Mouse tracheal epithelium was comparable to that reported in the literature with approximately $30 \%-35 \%$ of the surface consisting of ciliated respiratory epithelium imaged with SEM (7) (Figure 3A). This percentage is consistent with scanning electron micrographs of cultured tracheal epithelial cells at an air-liquid interface and previously published reports (6) (Figure 3B). On the other hand, the mouse septum had approximately $90 \%$ of the epithelial surface covered with cilia. (Figure 3C) This was consistent with the scanning electron micrograph of the air-liquid interface grown from dissociated mouse nasal septal epithelial cells at 17 days (Figure 3D).
Immunocytochemistry performed on the epithelial air-liquid interface at 17 days demonstrates an intact monolayer with tight junctions and a differentiated cell composition with cilia (Figure 4). Tight junctions are cell-to-cell adhesion structures in epithelial cells that constitute the epithelial junctional complex with adherens junctions and desmosomes. Tight junctions seal cells to create a primary barrier to the diffusion of solutes and function as a boundary between the apical and basolateral membrane domains to produce cellular polarization (8). Zo-1 is one of three major scaffold proteins concentrated at the cytoplasmic surfaces of the junctional complexes. Type IV $\beta$-tubulin is one of the major subtypes of $\beta$-tubulin in cilia and an excellent marker. Demonstration of Zo- 1 and type IV $\beta$-tubulin by immunocytochemistry indicates an intact polarized monolayer with a differentiated cell population.

Colocalization of type IV tubulin and OMP was performed to determine whether a significant proportion of the air-liquid interface culture was olfactory epithelial cells. OMP is a
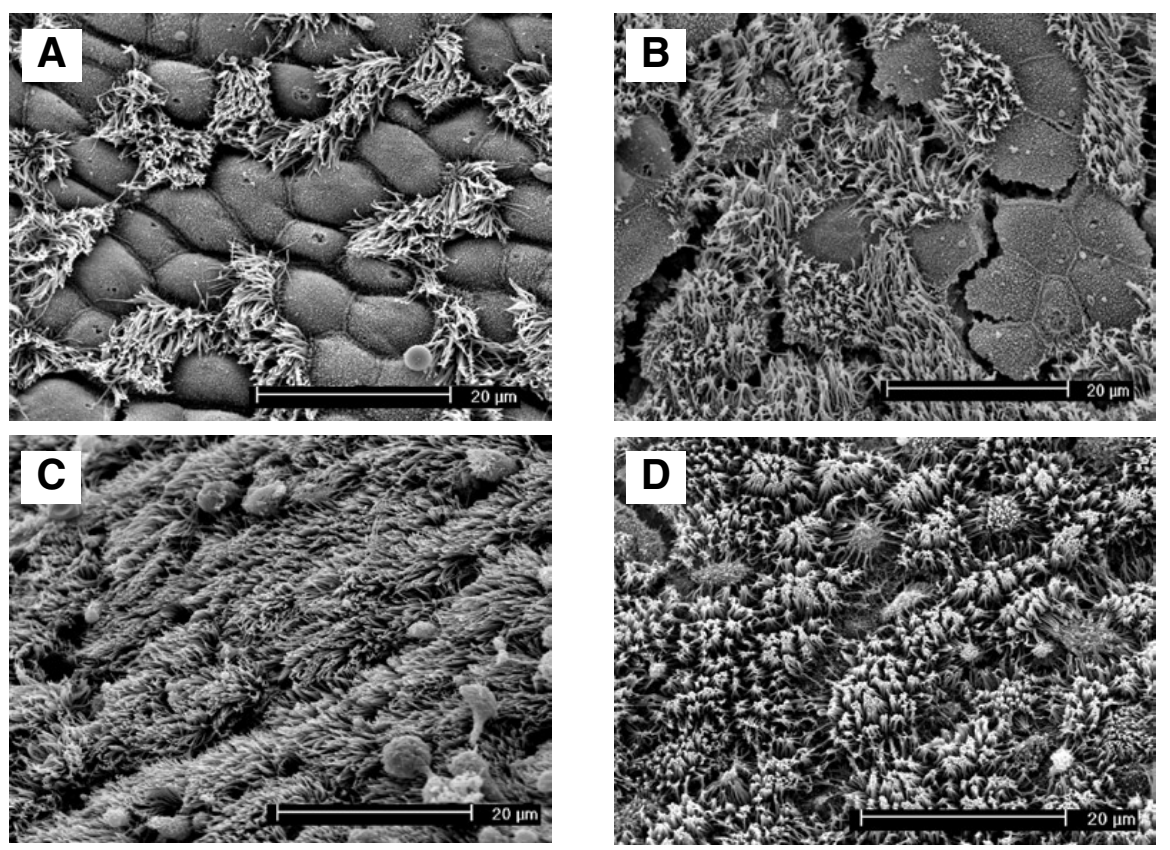

Figure 3. Morphologic comparison of in situ and culture of mouse trachea and nasal septum. (A) Scanning electron micrograph of a mouse trachea specimen. Only $35 \%$ ciliated respiratory epithelia are present, while the remainder are non-ciliated and clara cells. (B) The morphology of a tracheal epithelial air-liquid interface culture (fixed at 17 days) is very similar to native trachea. (C) This electron micrograph demonstrates the typical shag carpet of cilia present on the nasal septum. Approximately $90 \%$ of the surface has cilia. (D) Another scanning electron micrograph of an intact epithelial air-liquid interface from a mouse nasal septum demonstrates the high percentage of ciliated epithelial cells (17 days). 


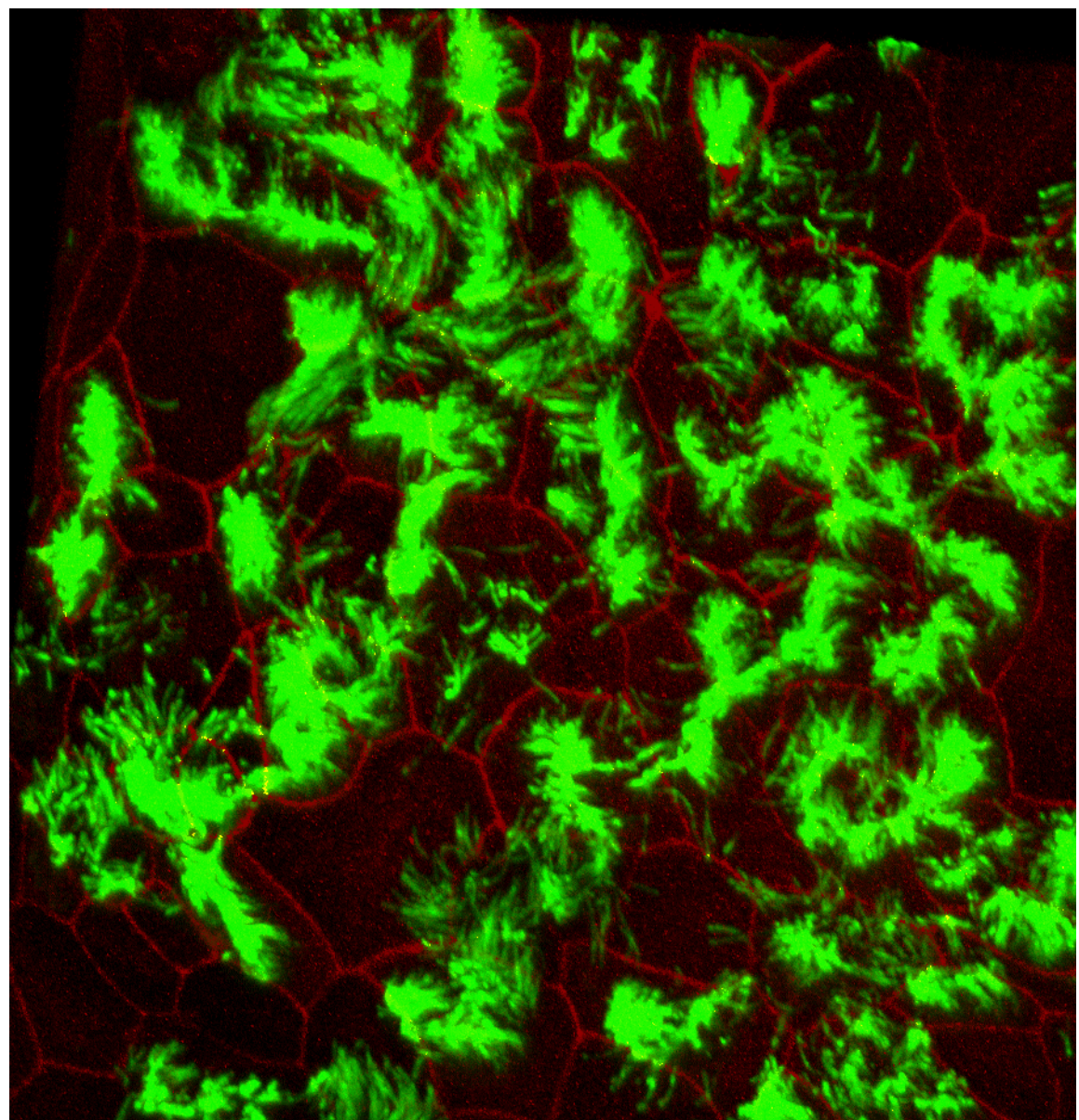

Figure 4. Type IV $\beta$-tubulin (green) staining for cilia and zona occludens-1 (Zo-1; red). Staining for tight junctions is demonstrated using confocal laser scanning microscopy $(63 \times)$ following immunocytochemistry of cultured epithelium on the air-liquid interface. This shows the green staining of the cilia at the top of the cell (differentiation) and the red staining of the tight junctions (confluence) between the cells of the monolayer.

inhibited by glybenclamide (mean inhibition 23\%). A representative tracing is shown in Figure 6.

The established techniques for the primary culture of airway epithelial cells from transgenic mouse tracheas have greatly facilitated the study of pulmonary diseases. Numerous investigators have described the maintenance of airway epithelial cells in a differentiated state in primary culture (6,9-11). However, the total number of cells that can be isolated from the mouse trachea is very small. The tracheal epithelium of the mouse has ciliated cells that occur only in scattered patches (7). In an attempt to increase the yield of tracheal epithelial cells, Kumar et al. (12) has recommended expansion of the population through serum-free growth media. However, the expansion and passage of murine respiratory epithelium has resulted in limited differentiation even after just one passage (1).

We have established a technique for the development of air-liquid interface cultures through the use of mouse nasal septa. Our technique of harvesting the nasal septum is simple and straightforward. This method increases the yield of respiratory epithelia 8-fold over tracheal cultures. The composition of murine nasal epithelium compares favorably to the nearly $90 \%$ ciliated respiratory epithelium we see in the developed air-liquid interface culture.

Septal air-liquid interface cultures exhibit full differentiation with cilia and confluence with tight junctions. OE, which comprises approximately half of the murine nasal septum, is absent on the differentiated air-liquid interface. The reasons for this are currently unknown, but likely due to the method of culture and absence of neuronal growth factors.

The presence of a resting $\mathrm{I}_{\mathrm{SC}}$ and of a resting electrical potential in these preparations is consistent with the presence of baseline net ion transport. The fact that the $\mathrm{I}_{\mathrm{SC}}$ was largely inhibited by amiloride suggests that at baseline these preparations are primarily sodium absorbing under short-circuit conditions. Furthermore, the presence of a forskolin-stimulated current that is partially inhibited by glybenclamide is strongly suggestive that these preparations are capable of secreting anions (most likely chloride) via the cystic fibrosis transmembrane

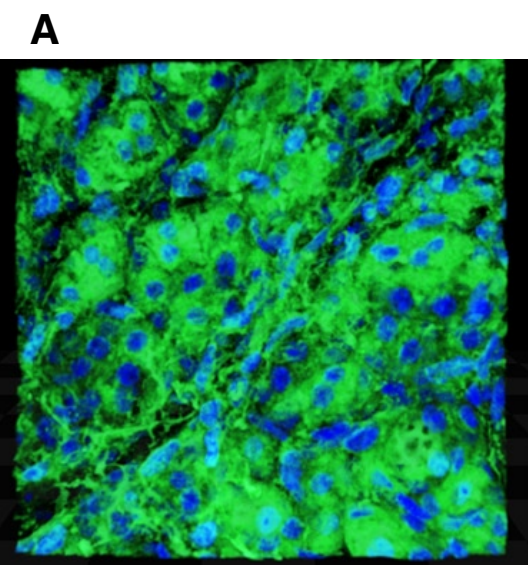

\section{B}

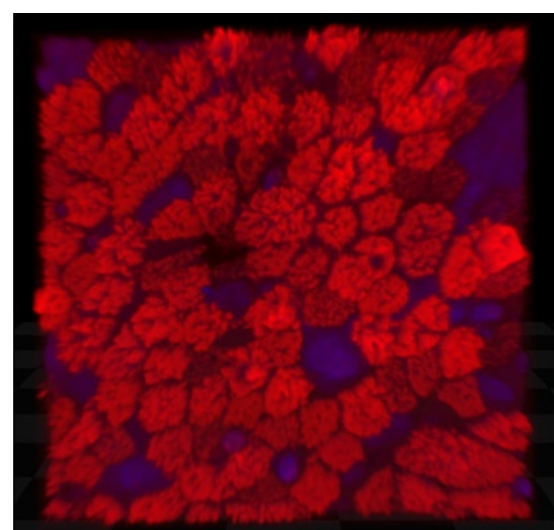

Figure 5. Olfactory marker protein (OMP) and type IV staining. (A and B) Immunofluorescence [septal olfactory epithelium (OE; panel A)] and immunofluorescence (septal air-liquid interface culture; panel B) for both OMP (green) and type IV $\beta$-tubulin (red). As expected, the septal OE stains heavily for OMP, while the air-liquid interface culture reveals staining only for type IV $\beta$-tubulin. Blue staining for nuclei is present [4',6-diamidino-2-phenylindole (DAPI)]. 


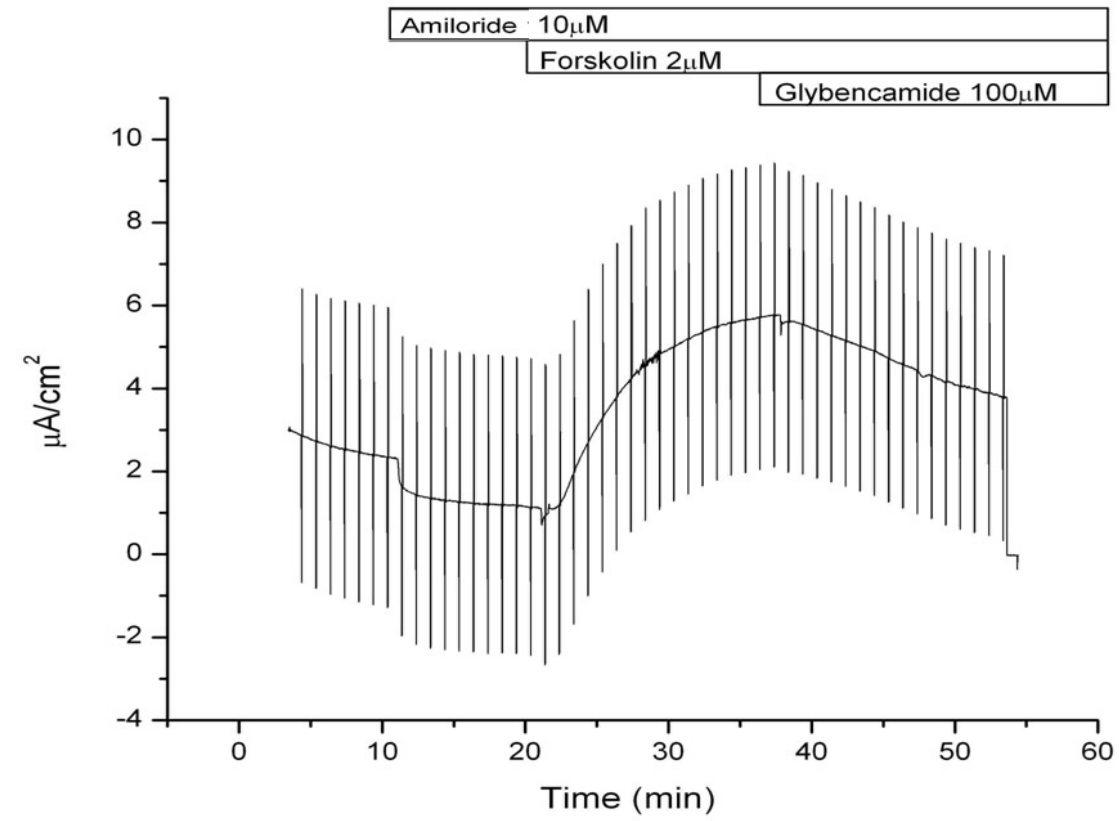

Figure 6. Representative short circiu current $\left(\mathrm{I}_{\mathrm{SC}}\right)$ tracing of polarized murine nasal epithelial cells. Murine nasal epithelial cells grown on Transwell permeable supports were mounted in Ussing chambers under short-circuit conditions and sequentially exposed to amiloride, forskolin, and glybenclamide. Note that baseline $\mathrm{I}_{\mathrm{SC}}$ is predominantly inhibited by amiloride and that forskolin-stimulated $\mathrm{I}_{\mathrm{SC}}$ is partially blocked by glybenclamide. These data are consistent with the presence of both electrogenic sodium absorption and anion secretion.

conductance regulator (CFTR)dependent mechanism.

The absolute value of our measured $\mathrm{I}_{\mathrm{SC}}$ is less than that calculated by others (13). This difference may be due to differences in mouse strain or age, or to differences in culture conditions. Also, our experiments were performed essentially at room temperature, which may have further contributed to this variation. Nevertheless, the overall phenotype of ion transport in these cells is consistent with an electrically active, mucociliary airway epithelium containing both a sodium-absorptive pathway and a chloride-secretory pathway $(14,15)$.

The increased yield and percentage of ciliated respiratory epithelium makes the murine nasal septa air-liquid interface model ideal for the study of sinonasal and pulmonary diseases and ciliary physiology. Air-liquid interface cultures grown from nasal septa exhibit a transepithelial potential. Thus, studying the growth and differentiation of septal respiratory epithelium will enable more physiologically relevant analysis of sinonasal and pulmonary diseases in vitro, and allow for the evaluation of novel therapies.

\section{ACKNOWLEDGMENTS}

M.B.A. and B.A.W. contributed equally to this work. This work was supported by the Young Investigator Award from the Sinus and Allergy Health Partnership (N.A.C.), and NIH grant nos. AI065450 (A.J.R.), K08HL081080 (J.L.K.), and R01 DK58046 (R.C.R.).

\section{COMPETING INTERESTS STATEMENT}

The authors declare no competing interests.

\section{REFERENCES}

1. You, Y., E.J. Richer, T. Huang, and S.L. Brody. 2002. Growth and differentiation of mouse tracheal epithelial cells: selection of a proliferative population. Am. J. Physiol. Lung Cell. Mol. Physiol. 283:L1315-L1321.

2.Doty, R.L. 1986. Odor-guided behavior in mammals. Experientia 42:257-271.

3. Halpern, M. and A. Martinez-Marcos. 2003. Structure and function of the vomeronasal system: an update. Prog. Neurobiol. 70:245-318.

4. Storan, M.J. and B. Key. 2006. Septal organ of Gruneberg is part of the olfactory system. J. Comp. Neurol. 494:834-844.
5.Benninger, M.S., B.J. Ferguson, J.A. Hadley, D.L. Hamilos, M. Jacobs, D.W. Kennedy, D.C. Lanza, B.F. Marple, et al. 2003. Adult chronic rhinosinusitis: definitions, diagnosis, epidemiology, and pathophysiology. Otolaryngol. Head Neck Surg. 129:S1-32.

6.Davidson, D.J., F.M. Kilanowski, S.H. Randell, D.N. Sheppard, and J.R. Dorin. 2000. A primary culture model of differentiated murine tracheal epithelium. Am. J. Physiol. Lung Cell. Mol. Physiol. 279:L766-L778.

7. Pack, R.J., L.H. Al-Ugaily, G. Morris, and J.G. Widdicombe. 1980. The distribution and structure of cells in the tracheal epithelium of the mouse. Cell Tissue Res. 208:65-84.

8. Gumbiner, B. 1987. Structure, biochemistry, and assembly of epithelial tight junctions. Am. J. Physiol. 253:C749-C758.

9.Davidson, D.J., M.A. Gray, F.M. Kilanowski, R. Tarran, S.H. Randell, D.N. Sheppard, B.E. Argent, and J.R. Dorin. 2004. Murine epithelial cells: isolation and culture. J. Cyst. Fibros. Suppl 2:59-62.

10. Davidson, D.J. and M. Rolfe. 2001. Mouse models of cystic fibrosis. Trends Genet. 17: S29-S37.

11. Lankford, S.M., M. Macchione, A.L. Crews, S.A. McKane, N.J. Akley, and L.D. Martin. 2005. Modeling the airway epithelium in allergic asthma: interleukin-13-induced effects in differentiated murine tracheal epithelial cells. In Vitro Cell. Dev. Biol. Anim. 41:217224.

12.Kumar, R.K., S.E. Maronese, and R. O'Grady. 1997. Serum-free culture of mouse tracheal epithelial cells. Exp. Lung Res. 23:427-440.

13. Grubb, B.R., R.N. Vick, and R.C. Boucher. 1994. Hyperabsorption of $\mathrm{Na}+$ and raised $\mathrm{Ca}(2+)$-mediated $\mathrm{Cl}$ - secretion in nasal epithelia of CF mice. Am. J. Physiol. 266:C1478C1483.

14. Adler, K.B., P.W. Cheng, and K.C. Kim. 1990. Characterization of guinea pig tracheal epithelial cells maintained in biphasic organotypic culture: cellular composition and biochemical analysis of released glycoconjugates. Am. J. Respir. Cell Mol. Biol. 2:145-154.

15. Gray, T.E., K. Guzman, C.W. Davis, L.H. Abdullah, and P. Nettesheim. 1996. Mucociliary differentiation of serially passaged normal human tracheobronchial epithelial cells. Am. J. Respir. Cell Mol. Biol. 14:104-112.
Received 9 December 2006; accepted 23 May 2007.

Address correspondence to Bradford A. Woodworth, Department of Otorhinolaryngology, 3400 Spruce Street, 5 Ravdin, Philadelphia, PA 19104, USA. e-mail: Bradford.Woodworth@uphs.upenn.edu

To purchase reprints of this article, contact: Reprints@BioTechniques.com 